Fernández, S.; Fernández-Río, J. (2019). Indoor-Cycling Practitioners' Motives and Addiction during a Year of Practice. Revista Internacional de Medicina y Ciencias de la Actividad Física y $\begin{array}{llllll}\text { el Deporte } & \text { vol. } & 19 & (76) & \text { pp. } & 673-683\end{array}$ Http://cdeporte.rediris.es/revista/revista76/artmotivos1101.htm

DOI: $10.15366 /$ rimcafd2019.76.008

\title{
ORIGINAL
}

\section{MOTIVOS DE PRÁCTICA Y ADICCIÓN EN PRACTICANTES DE CICLO-INDOOR A LO LARGO DE UN CURSO}

\section{INDOOR-CYCLING: PARTICIPANTS' MOTIVES AND ADDICTION DURING A YEAR OF PRACTICE}

\author{
Fernández, S. y Fernández-Río, J. \\ Doctores en Pedagogía. Facultad de Formación del Profesorado y Educación. Universidad de \\ Oviedo (España) U0194643@uniovi.es, javier.rio@uniovi.es
}

Código UNESCO / UNESCO code: 6105.09. Evaluación y Diagnóstico en Psicología. Validez de test / 6105.09. Evaluation and Diagnosis in Psychology. Test validity.

Clasificación Consejo de Europa / Council of Europe classification: 15. Psicología del Deporte / 15. Psychology of Sport.

Recibido 14 de febrero de 2018 Received February 14, 2018 Aceptado 1 de octubre de 2018 Accepted October 1, 2018

\section{RESUMEN}

El ciclo indoor es una de las actividades de fitness más practicadas, pero de la que apenas existen estudios científicos. El objetivo de esta investigación fue analizar el perfil motivacional de practicantes de ciclo indoor a lo largo de un curso entero. 379 practicantes (38.51 \pm 10.68 años; $64.6 \%$ mujeres, $35.4 \%$ varones) accedieron a participar. Al comienzo del curso (septiembre), a mitad (enero) y a finales (mayo), todos contestaron un cuestionario para medir los motivos de practica y los niveles de adicción al ejercicio. Los resultados mostraron que los motivos por los que realizaban dicha actividad variaron de manera significativa a lo largo del año: en septiembre y enero fitness/salud, mientras que en mayo apariencia. Respecto a su adicción al ejercicio, todos pueden ser catalogados como sintomáticos no dependientes; presentado un aumento significativo de la sintomatología en mayo. Ambos resultados parecen señalar hacia la conocida como "operación bikini".

PALABRAS CLAVE: Motivos. Adicción. Ciclo-indoor. Longitudinal. 


\begin{abstract}
Indoor cycling is one of the most practiced fitness activities. However, little research has been conducted. The goal of this study was to assess the motivational profile of indoor cycling participants throughout a whole year. 379 practitioners (38.51 \pm 10.68 years; $64.6 \%$ females, $35.4 \%$ males) agreed to participate. At the beginning of the course (September), in the middle (January) and at the end (May), all participants completed a questionnaire to assess motivation for physical activity and exercise addiction. Results showed that these indoor cycling participants' reasons to practice this activity changed significantly throughout the year: in September and January "fitness/health", and in May "appearance". Regarding exercise addition, this group of indoor cycling practitioners can be considered symptomatic non-dependent; their score significantly increased in may. Both results seem to point to the popularly known "bikini challenge".
\end{abstract}

KEY WORDS: Motives. Addiction. Indoor-cycling. Longitudinal.

\title{
1. INTRODUCCIÓN
}

La mayoría de los estudios realizados sobre la motivación hacia la práctica de actividad físico-deportiva se han centrado en contextos educativos o en ambientes deportivos competitivos; siendo muchos menos los enfocados a entornos deportivos no competitivos como los programas realizados en centros de fitness o gimnasios, tan populares en la actualidad (Sicilia, Águila, Muyor, Orta y Moreno, 2009). El Consejo Superior de Deportes (2010) señala que el perfil de práctica deportiva en España refleja una menor institucionalización (clubs, federaciones deportivas, asociaciones, organizaciones) y un alejamiento de la competición, ya que el $75 \%$ de las personas que realizan actividad física lo hacen al margen de cualquier entidad u organización.

Este hecho, junto a los importantes beneficios que se han asociado a la práctica de dichas actividades a nivel físico, psicológico y social (Taras, 2005) ha provocado que aparezcan más estudios sobre los motivos de inicio o mantenimiento de la práctica de actividad física en centros de fitness o gimnasios. Destacamos el realizado por Navarro et al. (2008) sobre practicantes de actividad física no-competitiva, que reveló dos perfiles de practicantes: (a) Autodeterminado: altas puntuaciones en regulación intrínseca e identificada, bajas en regulación externa y desmotivación y una práctica motivada por la diversión y los beneficios de la actividad (fitness/salud) y (b) No autodeterminado: altas puntuaciones en regulación externa y desmotivación, bajas en regulación intrínseca e identificada y una participación caracterizada por la búsqueda de incentivos externos. Moreno, Borges, Marcos, Sierra y Huéscar (2012) analizaron los intereses y motivaciones que mueven a las personas adultas a participar en actividades físico-deportivas en salas de fitness en función del tipo y frecuencia de práctica. Los resultados desvelaron la gran importancia que los participantes otorgan al motivo "salud" para realizar ejercicio físico, pero también destacaron los motivos "desarrollo de la habilidad" y "mejora de la imagen". Los 
practicantes de más de tres días a la semana puntuaron más alto en casi todos los motivos de práctica (imagen, afiliación y reconocimiento social) y tenían una mayor motivación integrada.

También se han estudiado los beneficios físicos, estéticos y psicológicos que conlleva la práctica regular y adecuada de ejercicio físico (Taras, 2005), que han sido reconocidos por la Organización Mundial de la Salud (2010). Sin embargo, lo que comienza como un hábito saludable puede convertirse en factor de riesgo o convertirse en un problema cuando se realiza en intensidades y frecuencias cuyas consecuencias son el deterioro y/o malestar, desarrollando conductas que van más allá de lo estrictamente necesario para mantener la forma física, y derivando en consecuencias físicas y/o psicológicas cuando la práctica del ejercicio es impedida o retirada. Se trata de dependencia al ejercicio y se estima que prevalece en torno al $3 \%$ de la población general, sufriendo un aumento cuando hablamos de los profesionales del mundo del deporte (Sussman, Lisha y Griffiths, 2011). El cómo y el por qué una persona pasa de ser sanamente comprometida con su práctica de ejercicio regular a ser adicta es explicado por varias teorías y todas coinciden en que es un trastorno de la conducta y se debe tanto a factores fisiológicos como psicológicos (Arbinaga y Caracuel, 2007). La mayoría de estudios sobre la adicción al ejercicio han sido realizados sobre actividades físicas específicas (corredores, fisicoculturistas...), siendo muy pocos los que se han centrado en usuarios de centros deportivos que practican diferentes actividades de fitness. González-Cutre y Sicilia (2012) analizaron las diferencias en dependencia del ejercicio según el sexo, la edad y la actividad física practicada en usuarios de centros de acondicionamiento físico (fitness) y obtuvieron que el $45.6 \%$ no presentaba síntomas de dependencia, el $47.4 \%$ mostraba síntomas, pero sin estar en riesgo y el $7 \%$ se podría considerar en riesgo de dependencia del ejercicio. Respecto al sexo, se observó que los hombres puntuaron más alto que las mujeres en los diferentes síntomas de la dependencia, observándose diferencias bastante significativas, excepto en abstinencia. Respecto a la edad, los resultados revelaron que según iba aumentando la dependencia esta iba disminuyendo. Finalmente, respecto a las actividades realizadas, estas se dividieron en actividades dirigidas, semidirigidas y libres y se observó que los usuarios que practicaban las dirigidas eran los que presentaban menor riesgo de dependencia.

En base a todo lo anterior, el objetivo de la presente investigación fue conocer si las motivaciones de un grupo amplio de practicantes de ciclo indoor por practicar este tipo de actividad física se mantienen estables a lo largo de un curso entero (septiembre-mayo). Las hipótesis planteadas fueron: a) Los practicantes de ciclo indoor tendrán siempre los mismos motivos para realizar dicha actividad física, independientemente de la época del año; b) El motivo predominante para hacer ciclo indoor será el disfrute de la misma; c) Los practicantes de ciclo indoor mostrarán un nivel de adicción al ejercicio físico de nivel bajo.

\section{MÉTODO}




\subsection{Participantes}

379 practicantes (38.51 \pm 10.68 años) de la actividad de fitness denominada ciclo indoor de una Comunidad Autónoma del norte de España accedieron a participar: $35.4 \%$ hombres y $64.6 \%$ mujeres, $53.3 \%$ realizaban dicha actividad en centros de titularidad pública frente al $46.7 \%$ que la realizaban en centros deportivos/gimnasios privados. Los requisitos de inclusión fueron: a) realizar ciclo indoor durante todo un curso completo (de septiembre a junio), b) completar como mínimo 2 sesiones semanales de ciclo indoor y c) acudir a más del $80 \%$ de las sesiones de práctica del año. Desafortunadamente, 32 personas no cumplieron los requisitos planteados por lo que tuvieron que abandonar el estudio; sus datos no se tuvieron en cuenta en el análisis posterior de los mismos.

\subsection{Instrumentos}

Escala de Medida de los Motivos para la Actividad Física-Revisada (MPAM-R; Ryan, Frederick, Lepes, Rubio y Sheldon, 1997). Se empleó para evaluar los motivos por los que los participantes realizaban ciclo-indoor. Se utilizó la versión validada al contexto español por Moreno, Cervelló y Martínez (2007). La escala original consta de 5 factores o sub-escalas, pero en esta investigación se usaron solo 4: Disfrute (7 ítems: i.e., "Porque es divertido"), Apariencia (6 ítems: i.e., "Porque quiero mantener mi peso para tener buena imagen"), Social (5 ítems: i.e., "Porque me gusta estar con mis amigos") y Fitness/salud (6 ítems: i.e., "Porque quiero estar en buena forma física). Al cuestionario se le cambió ligeramente la raíz original para acercarla más al objeto de estudio: "Realizo ciclo-indoor...". Los participantes respondieron en una escala tipo likert desde $1=$ "totalmente en desacuerdo" a 7= "totalmente de acuerdo". El análisis de los resultados de cada una de las subescalas mostró unos índices de fiabilidad (alpha de Cronbach) de $0.867,0.853,0.807$ y 0.855 , respectivamente.

Escala de Adicción al Ejercicio (EAl; Terry, Szabo y Griffiths, 2004). Se empleó para medir el grado de adicción de los participantes al ciclo-indoor. Se utilizó la versión validada al contexto español por Sicilia, Alías, Ferriz y Moreno (2013). Consta de un solo factor de 6 ítems (i.e., "El ejercicio es la cosa más importante de mi vida"). La escala va precedida de la raíz: "Hasta qué punto estás de acuerdo con los siguientes ítems....". Los participantes respondieron en una escala tipo Likert desde 1= "totalmente en desacuerdo" a $5=$ "totalmente de acuerdo". El cuestionario mostró un índice de fiabilidad (alpha de Cronbach) de 0.653. Este valor se sitúa ligeramente por debajo del mínimo exigido de 0.700 , pero el limitado número de ítems permite que sea usado (Vincent, 2005).

\subsection{Procedimiento}

En primer lugar, se solicitó la aprobación del comité de bioética de la Universidad de los investigadores. En segundo lugar, se contactó con todos los centros públicos y privados de la zona centro de la Comunidad Autónoma donde se desarrolló el estudio para obtener un listado de lugares donde se impartiera ciclo indoor. Se contactó con los responsables de cada centro, explicándoles el 
proyecto de investigación y solicitando su consentimiento para llevarlo a cabo. En aquellos en los que se obtuvo, se contactó con los monitores responsables para recabar también su aprobación. Obtenida esta se acudió en las horas y días concertados para explicar el proyecto a los practicantes de ciclo indoor, obteniendo el consentimiento informado de todos aquellos dispuestos a participar. Este consistía en recabar información de los practicantes de esta modalidad de actividad física (ciclo indoor) en tres momentos diferentes a lo largo de todo el año (curso completo). Finalmente, en septiembre, enero y mayo antes del comienzo de la actividad se acudió en las horas y días acordados y se pidió a los participantes que cumplimentaran el mismo cuestionario en los tres momentos. Se les aseguró la total confidencialidad de sus respuestas y que estas no tendrían repercusión sobre el monitor de la actividad. En ambos casos se buscaba que contestaran con total tranquilidad y honestidad.

\subsection{Análisis de datos}

Todos los análisis de los datos obtenidos se realizaron con el paquete estadístico SPSS versión 22.0 (IBM, Chicago, IL). En primer lugar, se procedió a comprobar la normalidad de los datos obtenidos. La prueba Kolmogorov-Smirnov $(p>.05)$ mostró que las variables a estudio seguían una distribución normal (Razali y Wah, 2011). Por lo tanto, a partir de ese momento se usaron pruebas paramétricas para el análisis de los datos. Se realizaron los pertinentes estadísticos descriptivos básicos (medias y desviación estándar) y de frecuencias. Posteriormente, se analizaron y compararon los datos obtenidos en los 3 momentos de extracción de datos seleccionados, septiembre, enero y mayo, a través de una Manova de medidas repetidas, obteniéndose también el tamaño del efecto de aquellas variables que presentaban diferencias significativas.

\section{RESULTADOS}

La tabla 1 muestra los resultados de los análisis estadísticos descriptivos básicos. Respecto a los motivos de práctica de ciclo indoor, estos participantes mostraron respuestas similares en los dos primeros momentos de extracción de datos (septiembre y enero). El motivo más importante era fitness/salud, seguido de disfrute, apariencia y social. Sin embargo, sus opiniones en mayo cambian y señalan en primer lugar apariencia, seguido de fitness/salud, disfrute y social.

Respecto al nivel de adicción de los participantes, señalar que presentaron niveles muy parejos en los dos primeros momentos de recogida de datos (septiembre y enero), pero mucho más altos en mayo. En los tres casos los niveles obtenidos indican que estos practicantes de ciclo indoor pueden ser considerados como individuos con síntomas de adicción.

Tabla 1. Medias y desviaciones estándar en cada mes de toma de datos.

\begin{tabular}{lccccccc}
\hline & \multicolumn{2}{c}{ Septiembre } & \multicolumn{2}{c}{ Enero } & \multicolumn{2}{c}{ Mayo } & \multirow{2}{*}{$f$} \\
\cline { 2 - 7 } & Media & DE & Media & DE & Media & DE & \\
\hline Disfrute & $5.40^{\mathrm{a}}$ & .95 & $5.30^{\mathrm{a}}$ & 1.06 & $4.43^{\mathrm{b}}$ & .95 & \\
\hline
\end{tabular}




\begin{tabular}{lccccccc} 
Apariencia & $4.47^{\mathrm{a}}$ & 1.12 & $4.55^{\mathrm{a}}$ & 1.2 & $5.84^{\mathrm{b}}$ & .91 & .527 \\
Social & $3.57^{\mathrm{a}}$ & 1.25 & $3.39^{\mathrm{a}}$ & 1.21 & $4.30^{\mathrm{b}}$ & 1.11 & .266 \\
Fitness/salud & $5.84^{\mathrm{a}}$ & .86 & $5.86^{\mathrm{a}}$ & .82 & $4.81^{\mathrm{b}}$ & .99 & .455 \\
Adicción al Ejercicio & $17.35^{\mathrm{a}}$ & 3.87 & $17.30^{\mathrm{a}}$ & 3.83 & $20.70^{\mathrm{b}}$ & 3.75 & .346 \\
\hline
\end{tabular}

Nota: Superíndices diferentes en una fila indican que existen diferencias significativas a nivel de $p>.005 ; f$ : tamaño del efecto.

La posterior Manova de medidas repetidas $3 \times 3$ (grupo, tiempo) nos permitió comprobar si existían diferencias significativas en función de la fecha de recogida de datos (septiembre, enero y mayo) sobre las distintas variables observadas, obteniéndose resultados positivos en varias de ellas. El análisis post-hoc de Bonferroni mostró que existían diferencias significativas en los motivos para la práctica de actividad física (en nuestro caso ciclo indoor): *Disfrute: Lambda de Wilks $=.605, \mathrm{~F}(2,339), \mathrm{p}<.001, \mathrm{\eta}^{2}=.394$, significativamente menor en mayo; *Apariencia: Lambda de Wilks $=.473, \mathrm{~F}(2,339), \mathrm{p}<.001, \eta^{2}=.527$, significativamente mayor en mayo; *Social: Lambda de Wilks $=.734 \mathrm{~F}(2,339)$, $\mathrm{p}<.001, \eta^{2}=.266$, significativamente mayor en mayo; $\mathrm{y}$ *Fitness/salud: Lambda de Wilks $=0.545, \mathrm{~F}(2,339), \mathrm{p}<.001, \eta^{2}=.455$, significativamente menor en mayo. También se obtuvieron resultados significativamente diferentes en la escala de Adicción: Lambda de Wilks = .654, F (2, 342), $\mathrm{p}<.001, \eta^{2}=.346$, significativamente mayor en mayo. Finalmente, se obtuvo el tamaño del efecto de la diferencia observada que puede considerarse grande en todos los casos (Vacha-Haase y Thompson, 2004). No se encontraron diferencias significativas ni en función del género, ni de la edad, ni del tipo de centro donde se realizaba la actividad (público o privado).

\section{DISCUSIÓN}

El objetivo fundamental de esta investigación fue conocer si las motivaciones de un grupo amplio de practicantes de ciclo indoor por realizar este tipo de actividad física se mantenían estables a lo largo de un curso entero de práctica, de septiembre a mayo. Los resultados obtenidos han mostrado que los motivos de práctica son similares en los dos primeros momentos del año (septiembre y enero): fitness/salud, seguido de disfrute, apariencia y social, pero su importancia cambió de manera significativa en mayo: apariencia, seguido de fitness/salud, disfrute y social. La misma tendencia se observó en el nivel de adicción: niveles muy parejos en los dos primeros momentos de recogida de datos (septiembre y enero), pero significativamente más altos en mayo. En los tres casos los niveles obtenidos indican que estos practicantes de ciclo indoor pueden ser considerados individuos con síntomas de adicción.

Nuestra primera hipótesis era que este grupo de practicantes de ciclo indoor tendrían siempre los mismos motivos para realizar dicha actividad física, independientemente de la época del año. Nuestros resultados han mostrado que esta hipótesis no se cumple en su totalidad, ya que mantuvieron estables los motivos de práctica durante la mayor parte del año, pero estos cambiaron al final del mismo. Por lo tanto, en mayo se produjo un cambio significativo en el tipo de motivo por el cuál este grupo de personas realizaban ciclo indoor. Desafortunadamente, no conocemos de estudios similares publicados que permitan comparar los resultados obtenidos. 
La segunda hipótesis planteada fue que el motivo predominante para hacer ciclo indoor sería el disfrute de la misma. Esta hipótesis no se cumplió en ningún caso. En septiembre y en enero el motivo más importante de práctica fue fitness/salud, seguido de disfrute, apariencia y social. Debido a que no existen estudios previos sobre los motivos que llevan a los usuarios a la práctica del ciclo indoor estos resultados solo pueden ser comparados con practicantes de otras actividades de fitness. De ellos destacamos el estudio realizado por Moreno et al. (2007) con practicantes de diferentes actividades físicas no competitivas (musculación, aerobic, actividades acuáticas, etc..) que encontraron el mismo orden de preferencia respecto a los motivos de práctica de dichas actividades que en los practicantes de ciclo indoor del presente estudio (salud, disfrute, apariencia y social). En otro estudio realizado por Moreno et al. (2012) también encontraron que los practicantes de actividades de fitness, tanto individuales como colectivas (i.e., musculación, pilates, aerobic, ciclo indoor, natación, fitness acuático) otorgaban gran importancia para su realización al motivo salud; datos también apoyados por otras investigaciones previas (García, 2006; Moreno, Cervelló, Borges y Conte, 2009; Navarro et al., 2008). Moreno et al. (2012) en el estudio anteriormente citado con usuarios de practicantes de actividades de fitness también encontraron puntuaciones altas en los motivos: "desarrollo de la habilidad" y "mejora de la imagen". Otros estudios a destacar son los realizados por Nuviala et al. (2014) en usuarios de centros deportivos y Moreno et al. (2016) en practicantes de actividades físicas no competitivas (musculación, aerobic, spinning, natación..). Los resultados obtenidos en ambos apoyan los resultados obtenidos en la presente investigación y que señalan al factor fitness/salud y disfrute como los más altamente señalados. Moreno et al. (2012) plantean que el disfrute es un predictor del motivo fitness/salud, ya que si una persona disfruta de la práctica de una actividad, seguirá practicándola.

El resultado más significativo de la presente investigación fue que el motivo de práctica de ciclo indoor de este grupo de personas cambió de manera significativa en la última toma de datos (mayo), siendo las puntuaciones más altas para la apariencia, seguida de fitness/salud, disfrute y social. Solamente hemos encontrado una referencia relacionada con la actividad física del ciclo indoor que apoye este resultado, en el cual la apariencia era el principal motivo de práctica de los usuarios de este tipo de actividad (Muyor y López, 2010). También han obtenido valores similares sobre la apariencia en un estudio realizado por Reche y Gómez (2014) en personas con trastorno de la conducta alimentaria y practicantes de actividad física, para los que el motivo principal por el que la realizaban era por la mejora de su aspecto físico, seguido del placer y de la mejora de la salud. La mayoría de estudios (Granero-Gallegos, GómezLópez, Abraldes y Rodríguez-Suárez, 2011; Nuviala et al., 2014) reflejan que la apariencia es el motivo menos valorado por los usuarios de los centros deportivos investigados. Una posible explicación al resultado encontrado en la presente investigación es que los usuarios de ciclo indoor en mayo estaban preocupados por su apariencia debido a la inminente llegada del verano, la llamada popularmente "operación bikini" (Córcoles, 2011). En la actualidad el valor que se le da al aspecto físico supera a muchos otros valores de la persona (Toro, 2013). Los medios de comunicación han contribuido a difundir el prototipo de belleza presente hoy en día, el femenino notablemente más delgado y el 
masculino más musculado. Esta preocupación por el aspecto físico, la autoimagen y la sobrevaloración del cuerpo, anteriormente mencionada, afecta tanto a los hombres como a las mujeres. Hoy en día esta preocupación por la apariencia a nivel social ha hecho incluso que a una determinada época del año se le denomine coloquialmente "operación bikini", a la cual ya todos los medios de comunicación hacen referencia. Se conoce como operación bikini al periodo previo al verano en las que muchas personas deciden perder peso, practicar más deporte y llevar una dieta con el fin de lucir el bañador o bikini presumiendo de una buena figura (Córcoles, 2011). Los resultados del presente estudio parecen confirmar que efectivamente esa "operación bikini" existe, y que nuestros practicantes de ciclo indoor se preocupan más por su apariencia en esta época del año cercana al verano (mayo).

Centrándonos en el factor social de práctica, en los tres momentos en los que los participantes cumplimentaron los cuestionarios (septiembre, enero y mayo), este factor obtuvo las puntuaciones más bajas. Estudios previos sobre practicantes de actividades de fitness (musculación, aerobic, ciclo indoor, fitness acuático, etc) encontraron resultados similares (García, 2006; Moreno et al. 2012). Por el contrario, otros estudios realizados con practicantes de actividades físicas no competitivas (Granero-Gallegos et al., 2011; Moreno, Águila y Borges, 2011) encontraron que el motivo social de práctica fue el más valorado. Asimismo, investigaciones en otros contextos deportivos, participantes de actividades competitivas, practicantes y competidores de deportes colectivos, revelaron que los adultos dan mayor importancia que los jóvenes a aspectos de relación social (Hodge, Allen y Smellie, 2008). En la presente investigación, aunque este motivo fue el menos valorado, podemos destacar que sufrió un aumento significativo en mayo.

Basándonos en las ideas de Navarro et al. (2008), cuyo objeto de estudio fue establecer perfiles motivacionales en la actividad física saludable desde la teoría de la autodeterminación, podemos decir que tanto en septiembre como en enero el perfil de nuestros participantes, practicantes de ciclo indoor, fue un perfil autodeterminado, ya que los motivos que priorizaron para su práctica fue fitness/salud y disfrute, mientras que en mayo mostraron un perfil menos autodeterminado, ya que el motivo principal fue apariencia. Como ya se ha señalado, la famosa "operación bikini" puede provocar un comportamiento menos autodeterminado y más influenciado por agentes externos a la propia persona. Los resultados obtenidos en la presente investigación así lo parecen indicar.

La tercera hipótesis de esta investigación fue que los practicantes de ciclo indoor de nuestro estudio mostrarían un nivel de adicción al ejercicio físico de nivel bajo. Los resultados mostraron que el nivel de adicción de estos participantes era medio y aumentó de manera significativa en mayo. En todos los momentos de recogida de información, estos practicantes de ciclo indoor obtuvieron un valor medio de adicción que los cataloga como sintomáticos no-dependientes; siendo esta sintomatología mayor en el último trimestre. Son pocos los estudios que se han centrado en la adicción dentro del ámbito de las actividades físicas no competitivas centradas en la salud, y ninguno si nos referimos a los practicantes de ciclo indoor. Uno de los pocos estudios con los que se pueden comparar 
nuestros resultados fue el realizado por González-Cutre y Sicilia (2012), los cuales analizaron las diferencias en dependencia del ejercicio según el sexo, la edad y las actividades físicas practicadas en usuarios de centros de acondicionamiento físico (fitness). Globalmente encontraron que el mayor porcentaje de usuarios mostraba síntomas de dependencia, pero sin estar en riesgo; tal y como se ha encontrado en la presente investigación. Estos datos también son apoyados por un estudio realizado con practicantes de actividades de fitness (Costa, Cuzzocrea, Hausenblas, Larcan y Oliva, 2012) y por otro estudio en el cual compararon sujetos practicantes de futbol con sujetos que hacían actividades de fitness (Lichtenstein, Larsen, Christiansen, Støving y Bredahl, 2014). En este último no se obtuvieron diferencias significativas en la prevalencia de la adicción entre estos grupos, por lo que se puede decir que ambos grupos plantean el mismo riesgo de presentar síntomas de adicción al ejercicio físico. En el estudio de Costa et al. (2012) se encontraron resultados similares a los obtenidos en nuestro estudio de ciclo indoor: el mayor porcentaje de practicantes estaban en el grupo de sintomáticos no dependientes.

Queremos volver a destacar los datos obtenidos en el mes de mayo, en el que el mayor motivo por el que los usuarios practicaban ciclo indoor fue la apariencia. Si revisamos los valores de la adicción al ejercicio, estos son también significativamente más altos que en septiembre y enero. Estos resultados corroboran los resultados obtenidos en trabajos anteriores (Sewell, Clough y Robertshaw, 1995) que afirman que las motivaciones por la imagen corporal juegan un papel muy importante en la aparición y mantenimiento de la adicción al ejercicio. Uno de los deportes que tiene una gran relación con la imagen corporal y con la adicción al ejercicio es el fisioculturismo; hasta tal punto que existe una patología denominada vigorexia o dismorfia muscular (Arbinaga y Carabel, 2007). Pierce y Morris (1998) también afirman que los practicantes de actividades de musculación presentan niveles de dependencia altos. En nuestros practicantes de ciclo indoor se ve reflejada en mayo la relación entre el motivo de apariencia para realizar dicha actividad y un aumento significativo de la adicción a su práctica.

\section{CONCLUSIONES}

A lo largo de un curso completo anual, los motivos por los que este grupo de participantes realizaba ciclo indoor cambiaron: en septiembre y enero el motivo principal fue fitness/salud, mientras que en mayo el motivo principal fue apariencia. Desde el punto de vista de su adicción al ejercicio físico, los resultados han mostrado que pueden este grupo de practicantes de ciclo indoor pueden ser catalogados como sintomáticos no dependientes, siendo la adicción más acentuada en mayo.

\section{REFERENCIAS BIBLIOGRÁFICAS}

Arbinaga, F. y Caracuel, J. C. (2007). Dependencia del ejercicio en fisicoculturistas competidores evaluada mediante la escala de Adicción General Ramón y Cajal. Universitas Psychologica, 6(3), 549-557. 
Consejo Superior de Deportes (2010). Encuesta sobre los hábitos deportivos en España. Madrid: Presidencia del Gobierno.

Córcoles, J. E. (2011). Estudio social de la población: El cuerpo en los gimnasios. Revista Digital Sociedad de la Información, 25, 1-16.

Costa, S., Hausenblas, H. A., Oliva, P., Cuzzocrea, F., \& Larcan, R. (2012). The role of age, gender, mood states and Exercise frequency on exercise dependence. Journal of Behavioral Addictions, 2(4), 216-223. https://doi.org/10.1556/JBA.2.2013.014

García, M. (2006). Posmodernidad y Deporte: Entre la individualización y la masificación. Encuesta sobre hábitos deportivos de los españoles 2005. Consejo Superior de Deportes-Centro de Investigaciones Sociológicas. Madrid.

González-Cutre, D. y Sicilia, Á. (2012). Dependencia del ejercicio físico en usuarios españoles de centros de acondicionamiento físico (fitness): diferencias según el sexo, la edad y las actividades practicadas. Psicología Conductual, 20(2), 349.

Granero-Gallegos, A., Gómez-López, M., Abraldes, J. A., y Rodríguez- Suárez, N. (2011). Motivos de práctica en el ámbito de la actividad física no competitiva. Espiral. Cuadernos del Profesorado, 4(7), 15-22. https://doi.org/10.25115/ecp.v4i7.915

Hodge, K., Allen, J. B., \& Smellie, L. (2008). Motivation in Master sport: Achievement and social goals. Psychology of Sport and Exercise, 9, 157176. https://doi.org/10.1016/i.psychsport.2007.03.002

Lichtenstein, M. B., Larsen, K. S., Christiansen, E., Støving, R. K., \& Bredahl, T. V. G. (2014). Exercise addiction in team sport and individual sport: prevalences and validation of the exercise addiction inventory. Addiction Research \& Theory, 22(5). https://doi.org/10.3109/16066359.2013.875537

Moreno, J. A., Cervelló, E., y Martínez Camacho, A. (2007). Validación de la escala de Medida de los Motivos para la Actividad Física-Revisada en españoles: Diferencias por motivos de participación. Anales de Psicología, 23(1), 167-176. https://doi.org/10.1037/t03515-000

Moreno, J. A., Cervello, E., Borges, F., y Conte, L. (2009). Importancia del interés por la opinión del practicante en la predicción del motivo fitness/salud en el ejercicio físico. Fitness \& Performance Journal, 8(4),247-253. https://doi.org/10.3900/fpj.8.4.247.s

Moreno, J. A., Martínez Galindo, C., González-Cutre, D., y Marcos, P. (2009). Perfiles motivacionales de practicantes en el medio acuático frente al medio terrestre. Revista Internacional de Medicina y Ciencias de la Actividad Física y el Deporte, 9(34),201-216.

Moreno, J. A., Águila, C., y Borges, F. (2011). La socialización en la práctica físico-deportiva de carácter recreativo: predictores de los motivos sociales. Apunts. Educació Física i Esports, 103, 76-82.

Moreno, J. A., Borges, F., Marcos, P. J., Sierra, A. C., y Huéscar, E. (2012). Motivación, frecuencia y tipo de actividad en practicantes de ejercicio físico. Revista Internacional de Medicina y Ciencias de la Actividad Física y el Deporte, 12(48), 649-662.

Muyor, J. M., y López-Miñarro, P. A. (2010) Perfil de los usuarios que acuden a un centro especializado de Ciclo Indoor. En: I Jornadas Andaluzas de la industria del fitness. Salud y gestión en un mismo sector. Sevilla. 
Navarro, N., González-Cutre, D., Marcos, P. J., Borges, F., Hernández, A., Vera, J. A., y Moreno, J. A. (2008). Perfiles motivacionales en la actividad física saludable: un estudio desde la perspectiva de la teoría de la autodeterminación. En Actas del XI Congreso Nacional, XI Andaluz y III Iberoamericano de Psicología de la Actividad Física y del Deporte. Sevilla: Universidad Pablo de Olavide.

Nuviala, R. N., Teva-Villén, M. R., Pérez-Ordás, R., Grao-Cruces, A., Fajardo, J. A. T., y Nuviala, A. N. (2014). Segmentación de usuarios de servicios deportivos. Retos, 25, 90-94.

Organización Mundial de la Salud (2010). Recomendaciones mundiales sobre actividad física para la salud.

Pierce, E. F., \& Morris, J. T. (1998). Exercise dependence among competitive power lifters. Perceptual and Motor Skills, 86, 1097-1098. https://doi.org/10.2466/pms.1998.86.3.1097

Razali, N. M., \& Wah, Y. B. (2011). Power comparisons of Shapiro-Wilk, Kolmogorov-Smirnov. Lilliefors and Anderson-Darling tests. Journal of Statistical Modeling and Analytics, 2(1), 21-33.

Reche, C., y Gómez, M. (2014). Dependencia al ejercicio físico y trastornos de la conducta alimentaria. Apuntes de Psicología, 32(1), 25-32.

Ryan, R. M., Frederick, C. M., Lepes, D., Rubio, N., \& Sheldon, K. M. (1997). Intrinsic motivation and exercise adherence. International Journal of Sport Psychology, 28, 335-354.

Sewell, D. F., Clough, P. J., \& Robertshaw, L. (1995). Exercise addiction, mood and body image: A complex inter-relationship. En J. Annett, B. Cripps y $\mathrm{H}$. Steinberg (Eds.), Exercise addiction: Motivation for participation in sport and exercise (pp. 34-39). Leicester: British Psychological Society.

Sicilia, A., Águila, C., Muyor, J. M., Orta, A., y Moreno, J. A. (2009). Perfiles motivacionales de los usuarios en centros deportivos municipales. Anales de Psicología, 25(1), 160-168

Sicilia, Á., Alías, A., Ferriz, R., \& Moreno, J. A. (2013). Spanish adaptation and validation of the Exercise addiction inventory (EAI). Psicothema, 25(3), 377383.

Sussman, S., Lisha, N., \& Griffiths, M. (2011). Prevalence of the addictions: A problem of the majority or the minority? Evaluation \& the Health Professions, 34(1), 3-56. https://doi.org/10.1177/0163278710380124

Taras, H. (2005). Physical activity and student performance at school. The Journal of School Health, 75(6), 214-218. https://doi.org/10.1111/j.17461561.2005.00026.x

Terry, A., Szabo, A., \& Griffiths, M. (2004). The exercise Addiction inventory: A new brief screening tool. Addiction Research and Theory, 12(5), 489-499. https://doi.org/10.1080/16066350310001637363

Toro, J. (2013). El adolescente ante su cuerpo. Cuerpo, vestido y sexo. Madrid: Ediciones Pirámide (Grupo Anaya, S.A)

Vacha-Haase, T., \& Thompson, B. (2004). How to estimate and interpret various effect sizes. Journal of Counseling Psychology, 51(4), 473-481. https://doi.org/10.1037/0022-0167.51.4.473

Vincent, W. (2005). Statistics in Kinesiology. Champaign, IL: Human Kinetics. 
Rev.int.med.cienc.act.fís.deporte - vol. 19 - número 76 - ISSN: 1577-0354

Número de citas totales / Total references: 31 (100\%)

Número de citas propias de la revista / Journal's own references: 2 (6,4\%)

Rev.int.med.cienc.act.fís.deporte - vol. 19 - número 76 - ISSN: 1577-0354 